International Journal of Modern Physics D

(C) World Scientific Publishing Company

\title{
Peculiar velocity measurement in a clumpy universe
}

\author{
FARHANG HABIBI \\ $L A L, I N 2 P 3-C N R S$, \\ B.P. 34, 91898 Orsay Cedex, France \\ habibi@lal.in2p3.fr \\ SHANT BAGHRAM \\ Department of Physics, Sharif University of Technology, \\ P. O. Box 11155-9161, Tehran, Iran \\ baghram-AT-sharif.edu \\ SAEED TAVASOLI \\ Department of Physics, Kharazmi University, \\ P. O. Box 31979-37551, Tehran, Iran \\ tavasoli@ipm.ir
}

\begin{abstract}
Aims: In this work we address the issue of peculiar velocity measurement in a perturbed Friedmann universe using the deviations from measured luminosity distances of standard candles from background FRW universe. We want to show and quantify the statement that in intermediate redshifts $(0.5<z<2)$, deviations from the background FRW model are not uniquely governed by peculiar velocities. Luminosity distances are modified by gravitational lensing. We also want to indicate the importance of relativistic calculations for peculiar velocity measurement at all redshifts.

Methods: For this task we discuss the relativistic correction on luminosity distance and redshift measurement and show the contribution of each of the corrections as lensing term, peculiar velocity of the source and Sachs-Wolfe effect. Then we use the SNe Ia sample of Union 2, to investigate the relativistic effects we consider.

Results: we show that, using the conventional peculiar velocity method, that ignores the lensing effect, will result in an overestimate of the measured peculiar velocities at intermediate redshifts. Here we quantify this effect. We show that at low redshifts the lensing effect is negligible compare to the effect of peculiar velocity. From the observational point of view, we show that the uncertainties on luminosity of the present $\mathrm{SNe}$ Ia data prevent us from precise measuring the peculiar velocities even at low redshifts $(z<0.2)$.
\end{abstract}

\section{Inroduction}

The propagation of the light from standard candles, such as supernovae type Ia (SNe Ia), from the source to the observer is of greatest interest for cosmology. On one hand, standard candles are used to measure the rate of the expansion of the universe $\frac{12}{2}$ On the the other hand, the amount of light bundle distortion of the source is used to measure the gravitational effect of the matter distribution along the line of sight, which helps us to learn about the distribution and evolution of 
the matter in different cosmic times and scales $\frac{3.4}{4}$ Another phenomenon which has an important effect on the measurement of the magnitude of the standard candle and its interpretation is the peculiar velocity. This is because the peculiar velocity changes the redshift of the source ${ }^{5}$ The host galaxies of standard candles has an additional velocity beside the Hubble expansion velocity. Although the existence of peculiar velocity makes ambiguity in the measurement of the cosmological redshift, however it is used as a promising tool to probe the matter distribution, this is because the peculiar velocity of matter is sourced by the change in the matter perturbation due to continuity equation. Accordingly the peculiar velocity of luminous matter (i.e. galaxies) can be used as a tracer of the dark matter distribution in the Universe. As future large-scale structure surveys will open up a great opportunity to map the Universe on larger scales and at deeper redshifts, peculiar velocity measurements, lensing maps of the Universe and distance measurements of standard candles become progressively more important ${ }^{6}$ These observations can be used as a probe to check the standard model of cosmology or to see any deviations from $\Lambda \mathrm{CDM}]^{7-10}$ Accordingly, the accurate measurement of these quantities, their hidden relations and their model dependencies have great importance. Historically, the peculiar velocities are obtained by the relation $v_{p}=c z-H r$, where $v_{p}$ is the peculiar velocity, $z$ is the observed redshift, $H$ is the value of Hubble parameter and $r$ is the physical distance to the source respectively and $\bar{v}=H r$ is the velocity of the Hubble flow 1118 Using the peculiar velocity relation one can measure the distances to the sources and having the observed redshifts, the peculiar velocity can be deduced. However, distance measurement is a non-trivial exercise and there are many different methods to do this. The Tully-Fisher relation for spirals, ${ }^{19}$ surface brightness fluctuations for nearby galaxies, ${ }^{20}$ the Faber-Jackson relation for elliptical galaxies, ${ }^{21}$ the tip of the Red Giant Branch, Cepheids, and SNe Ia are the most developed methods to measure the distances ${ }^{22}$ In this work, first we reexamine the luminosity distance measurements in a clumpy universe by accounting for the lensing, 23]26 Sachs-Wolfe and Integrated Sachs-Wolfe effects. ${ }^{27}$ Then we demonstrate the relativistic approach to measuring peculiar velocities similar to the one presented in Davis and Scrimgeour, $\stackrel{28}{2}$ and finally we take into account both relativistic corrections and lensing corrections to luminosity distance computation in $\Lambda \mathrm{CDM}$ framework. In this work, we also study the physics of the peculiar velocity measurement, where we show that the distance measurement methods which assume that the observed distance to a host galaxy is equal to its proper distance are all biased. This bias comes from the fact that deviation from unperturbed FRW Universe is not considered in distance measurement. In other words, the over/under dense regions along the line of sight affect the luminosity of the standard candle. Accordingly we assert that the distance-independent measurements, like peculiar velocity measurements by redshift-space distortions, linear theory or kinetic Sunyaev-Zeldovich effect are more unbiased observation to find the peculiar velocity. Although with the nowadays data, we currently have larger errors using these methods, $\frac{29}{29}$ the results of this study will be important to be applied in future Large Scale Structure (LSS) surveys, which probe the Universe in higher redshifts, where the unbiased measurement of the distances become important. It is very essential to note that the full relativistic treatment of the peculiar density measurements can be done by 
the use of spacetimes equipped with two families of observers,(one which is fixed with CMB reference frame and the other which follow the matter (like a galaxy) flow) with their associated 4-velocities forming a "tilt angle" 30 In this direction there are studies that shows how peculiar velocities could change the way observers interpret their cosmological data ${ }^{31}$ However in this work we do not use the tilted coordinates, which can be an idea for extending this study. The structure of this work is as follow: In the first subsection of Sec. 22 we study the Luminosity distance in clumpy Universe without peculiar velocity. In the second subsection we study the case of peculiar velocity as a redshift redefinition parameter and in the third subsection we take into account the physics of both peculiar velocity and gravitational lensing. In Sec. 3 we have the conclusion and future prospects.

\section{Luminosity distance}

In a homogenous-isotropic universe with FRW metric:

$$
d s^{2}=-d t^{2}+a^{2}(t) d \sigma^{2}
$$

where $d \sigma^{2}=d \chi^{2}+f_{K}^{2}(\chi)\left[d \theta^{2}+\sin ^{2} \theta d \phi^{2}\right]$ is the spatial line element and $f_{K}$ is:

$$
f_{K}(\chi)= \begin{cases}\sin \chi & \text { if } K=+1 \\ \chi & \text { if } K=0 \\ \sinh \chi & \text { if } K=-1\end{cases}
$$

where $\chi$ is the comoving distance and $K$ is the space curvature. We can measure the cosmological parameters by measuring the luminosity distance of the standard candles such as SNe Ia. The luminosity distance $d_{L}$ is defined as the ratio of the absolute luminosity $L_{s}$ of the source to the observed flux $\mathcal{F}$, by:

$$
d_{L}^{2}=\frac{L_{s}}{4 \pi \mathcal{F}} .
$$

The luminosity distance in the flat-FRW model is related to comoving distance by:

$$
\bar{d}_{L}(\bar{z})=(1+\bar{z}) \chi(\bar{z})=(1+\bar{z}) \int_{0}^{\bar{z}} \frac{c d z^{\prime}}{H\left(z^{\prime}\right)},
$$

where $\bar{d}_{L}(\bar{z})$ is the background luminosity distance (luminosity distance in the unperturbed universe described by FRW metric) at background (or cosmological) redshift $\bar{z}$. Note that hereafter ${ }^{-}$indicates the background quantity. The background luminosity distance is related to the distance modulus by:

$$
\bar{\mu}=m-M=5 \log \left(\bar{d}_{L}(\bar{z})\right)+25,
$$

where $m$ and $M$ are the apparent and absolute magnitudes of a standard candle (such as SNe Ia) and in this case $d_{L}$ is measured in Mpc units and $\mu$ can be interpreted as a distance indicator (amount of magnitude change), interchangeably.

In the first subsection, we study the distance measurement case, in a clumpy universe with change in the background redshift. In the second subsection, we study the case of unperturbed Universe with the inclusion of the effects that change the observed background redshift like peculiar velocity. This will be done in relativistic manner and finally in the third subsection we study both the effect of peculiar velocity and convergence on the luminosity distance measurement. 


\subsection{Luminosity distance in a clumpy universe with zero peculiar velocities}

The Universe is not exactly homogenous and isotropic. The cosmic structures include over-dense (galaxies, groups and clusters of galaxies) and under-dense (voids) regions which makes the Universe to differ from being a FRW space-time. Hence, we should consider a perturbed metric for the clumpy Universe in order to investigate the propagation of light through voids and over-dense regions. We use the perturbed FRW-metric in Newtonian gauge:

$$
d s^{2}=-(1+2 \Psi(\vec{x}, t)) c^{2} d t^{2}+a^{2}(t)(1-2 \Phi(\vec{x}, t)) d \sigma^{2},
$$

where $\Psi(\vec{x}, t)$ and $\Phi(\vec{x}, t)$ are the perturbed scalars. In the case that we have the general relativity as the classical theory of gravity and the assumption that the cosmic fluid does not have an anisotropic terms in its energy-momentum tensor, we have $\Psi=\Phi$.

In order to study the effect of inhomogeneities on the propagation of the light bundle we have to solve the Sachs equation!

$$
\frac{d^{2}}{d \chi^{2}} d_{L}=-\frac{1}{2} R_{\mu \nu} k^{\mu} k^{\nu} d_{L}
$$

where we replaced the angular diameter distance with luminosity distance as in metric theories we have $d_{L}=(1+z)^{2} d_{A}$. $R_{\mu \nu}$ is the Ricci tensor and $k^{\mu}$ is the four momentum of photon which is defined by using the background comoving distance as the affine parameter $k^{\mu}=d x^{\mu} / d \chi$. Using the Sachs equation the luminosity distance in perturbed universe $d_{L}(\bar{z})$ becomes:

$$
d_{L}(\bar{z})=\bar{d}_{L}(\bar{z})\left[1+\frac{\delta d_{L}}{\bar{d}_{L}(\bar{z})}\right]
$$

where $\delta d_{L} / \bar{d}_{L}(\bar{z})$ is the change of the luminosity distance with respect to the FRW prediction, due to effect of structures along the line of sight. The $\delta d_{L} / \bar{d}_{L}(\bar{z})$ is as below!27

$$
\left.\frac{\delta d_{L}}{\bar{d}_{L}}\right|_{\chi_{s}}=\hat{n} \cdot \vec{v}_{0} / c-\Phi_{s}+\frac{1}{\chi_{s}} \int_{0}^{\chi_{s}} d \chi_{s}\left(2 \Phi-\left(\chi-\chi_{s}\right) \chi \nabla_{\perp}^{2} \Phi\right),
$$

where $\vec{v}_{0}$ is the peculiar velocity of the observer, $\hat{n}$ is the unit vector from the observer toward the source, $\Phi_{s}$ is the gravitational potential of the host and $\chi_{s}$ is the comoving distance to source respectively. The 2 dimensional Laplacian is defied as $\left(\nabla_{\perp}^{2}=\nabla^{2}-(\hat{n} . \vec{\nabla})^{2}+2 \hat{n} . \vec{\nabla} / \chi\right)$. It is worth to mention that there is no term related to the peculiar velocity of source in luminosity distance change obtained Eq. 9 ). The other important thing to indicate is that a crucial term in the luminosity distance change is the lensing convergence $\kappa_{g}$ term, which is defined from the second term of the integral in Eq. (9) as:

$$
\begin{aligned}
\kappa_{g} & =\int_{0}^{\chi_{s}} d \chi\left(\chi_{s}-\chi\right) \frac{\chi}{\chi_{s}} \nabla_{\perp}^{2} \Phi \\
& \simeq \frac{3}{2} H_{0}^{2} \Omega_{m} \int_{0}^{\chi_{s}} d \chi\left(\chi_{s}-\chi\right) \frac{\chi}{\chi_{s}}\left(1+z_{s}\right) \delta_{m}(\chi),
\end{aligned}
$$


where the second equation is an approximation because we exchange the 2D Laplacian with that in $3 \mathrm{D}$. We use the Poisson equation to replace the $\Phi$ with density contrast $\delta_{m}$, which depends on the comoving distance. $\Omega_{m}$ is the matter density parameter in the present time.

In the case that we can neglect the potential terms with respect to the convergence term, the luminosity distance of the standard candles will be modified as below:

$$
d_{L}(\bar{z})=\bar{d}_{L}(\bar{z})\left[1-\kappa_{g}(\bar{z})\right],
$$

where $d_{L}(\bar{z})$ is the observed luminosity distance at comoving redshift, $\bar{z}$, produced by the Hubble expansion. The $\kappa_{g}$ is the gravitational lensing correction obtained from the line of sight integration of the matter density contrast as in Eq. 10$].{ }^{3}$ We should note that gravitational lensing only change the source apparent magnitude and do not affect the redshift of the source. Therefore, in the absence of peculiar velocities, the observed redshift is the same as the comoving redshift: $z=\bar{z}$.

An important example of a host galaxy with no peculiar velocity is the one that resides at the center of a cosmic void or center of a galaxy cluster (a Brightest Central Galaxy (BCG)).

In addition, in relation (9), there are two gravitational potential terms other than $\kappa_{g}$ that can affect the observed luminosity. In the next section, we will include the effect of gravitational potential change on the source luminosity and will show that at low and intermediate redshifts this effect is negligible.

\subsection{Luminosity distance with peculiar velocity as an example of redshift redefinition}

At low redshifts $(z<0.1)$, the line-of-sight effects do not significantly affect the light bundle of the standard candles. In order to confirm this assertion, we consider the fact that the variance of the $\kappa_{g}$ is related to the matter power spectrum via Eq. (10). We plot this theoretical variance of $\kappa_{g}$ versus redshift in Fig. 1. According to the figure, at low redshifts $\kappa_{g} \sim 10^{-3}$. By using relation 11), this creates a typical distance modulus fluctuation of $\sim 10^{-3}$ magnitude. As we will see in this section, this fluctuation is negligible in comparison to the fluctuation produces by peculiar velocity which is at order of 0.1 magnitude. The peculiar velocities of the sources, produced by the local density contrast (as in the case of a SNe Ia host galaxy which resides in in-falling regions in a galaxy group ), can have a dominant effect on the luminosity distance computed by background $\Lambda \mathrm{CDM}$ model through changing the redshift from $\bar{z}$ to $z$. This redshift distortion can be corrected to reproduce the background model by Taylor expansion of Eq.(4) around the observed redshift $z$ :

$$
\bar{d}_{L}(\bar{z})=\bar{d}_{L}(z)+\left.\frac{\partial \bar{d}_{L}}{\partial \bar{z}}\right|_{z}(\bar{z}-z) .
$$

The non-zero $\bar{z}-z$ is produced by the peculiar velocity of the source. However in order to take all the terms that contribute to the redshift redefinition, we have to solve the volume expansion equation $\underline{\underline{3}}$

$$
\frac{d z}{d \chi}=\left[-\frac{\Theta}{3}+\sigma_{\mu \nu} n^{\mu} n^{\nu}\right](1+z)^{2}
$$




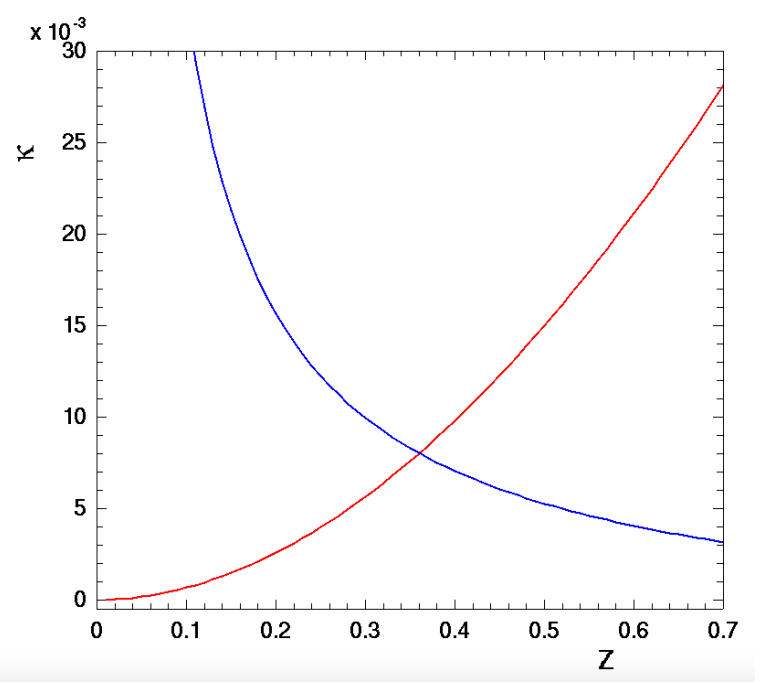

Fig. 1. Red curve shows the change of the theoretical variance of $\kappa_{g}$ versus redshift. Blue curve represents the variation of $\kappa_{v}$ for $v_{p}=1000 \mathrm{~km} / \mathrm{s}$ as a function of redshift. Gravitational lensing stars dominating the peculiar velocity after $z \sim 0.35$. The curves are plotted for a flat $\Lambda \mathrm{CDM}$ universe with $\Omega_{m}=0.27$ and $H_{0}=70 \mathrm{~km} / \mathrm{s} / \mathrm{Mpc}$.

where $\Theta$ is the expansion parameter, $\sigma_{\mu \nu}$ is anisotropic stress and $n^{\mu}$ is normal 4-vectors. Solving the Eq. 13) we will get:

$$
\frac{z-\bar{z}}{1+\bar{z}}=z_{p}-z_{S W} / 2-2 \int_{0}^{\chi_{s}} d \chi \Phi^{\prime}
$$

where $z_{p}$ is the peculiar redshift due to the peculiar velocity of the source and $z_{S W}=$ $2\left(\Phi_{s}-\Phi_{0}\right)$ is the redshift induced by Sachs-Wolf effect. The gravitational potential can be estimated as $\Phi \sim\left(v_{s} / c\right)^{2}$ where $v$ is the dispersion velocity of the source host galaxy. At intermediate and low redshifts $z_{S W} \ll z_{p}$.

Neglecting the gravitational potential contribution, we focus our attention to the peculiar velocity as the main source of the redshift deviation from the background redshift. Using the relativistic peculiar redshift, we have:

$$
1+z_{p}=\sqrt{\frac{1+\left(v_{p} / c\right)}{1-\left(v_{p} / c\right)},}
$$

where $c$ is the light speed. $v_{p}$ is the peculiar velocity along the line of sight which is related to the source proper motion through relation $v_{p}=\hat{n} . \vec{v}_{s}$, where $\hat{n}$ is the unit vector pointing from the observer to the source. One should note that the equation above is valid for measuring any relativistic velocity. In order to formulate the $v_{p}$, we start with the definition of physical distance $r=a \chi$, where $a=1 /(1+z)$ is the scale factor. We neglectthe gravitational potential corrections on proper distance measurement. Consequently, the observed velocity in non-relativistic limit is written 


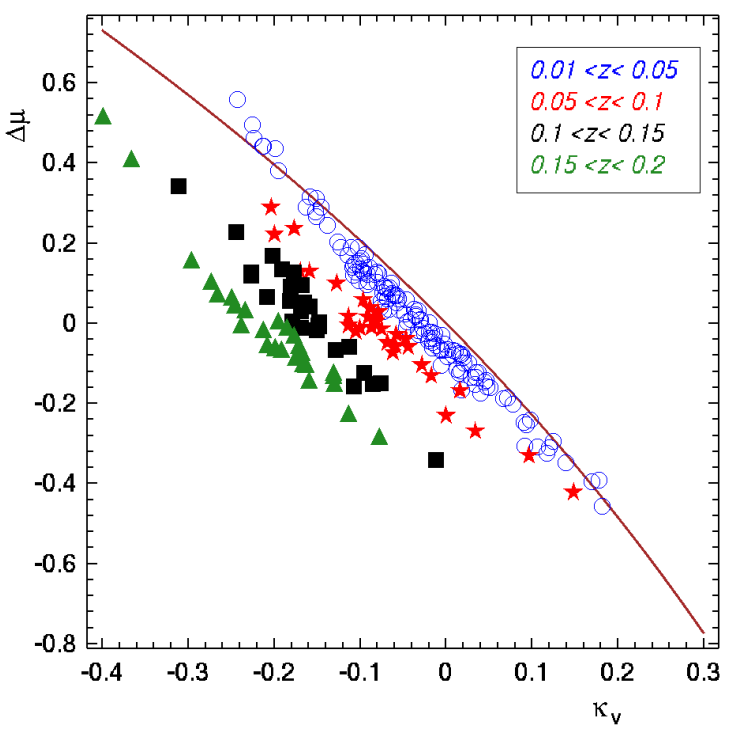

Fig. 2. The solid curve represents relation 23). The symbols show peculiar redshifts of $\mathrm{SNe}$ computed non-relativistically through relation (18) and substituted in $\kappa_{v}$ relation. The deviation from the relativistic curve increases by redshift.

as:

$$
v=\bar{v}+v_{p}
$$

where $\bar{v}$ is the cosmological Hubble velocity $(\bar{v}=H r)$. In order to relate the peculiar velocity $v_{p}$ to the observed redshift $z$ and the unperturbed cosmological redshift $\bar{z}$, we use the relativistic relation 28

$$
v=c \frac{(1+z)^{2}-1}{(1+z)^{2}+1},
$$

which in the non-relativistic limit reduces to $v \simeq c z$ and gives the well known but approximate relation:

$$
v_{p} \simeq c z-H d_{L}(z)
$$

where we have approximated $r(z)$ by the luminosity distance $d_{L}(z)$. In, 28 they show that the relation $v \simeq c z$ introduces accountable errors even at low redshift. The correct relation between the redshifts is as below:

$$
(1+z)=(1+\bar{z})\left(1+z_{p}\right)
$$

The relation $z \simeq \bar{z}+z_{p}$ works only at low redshifts and small peculiar velocities. The equation 19 can be derived by considering how two different inertial observers, located at any co-moving distance from each other on the Hubble flow, measure the wavelength emitted by a source with a peculiar motion. The important task now is to find the peculiar velocity (or equivalently the peculiar redshift $z_{p}$ ). By computing 
the derivative of equation (4) and substituting $\bar{z}-z$ from equation (14) (where we neglect the potential related terms), the equation (12 becomes as:

$$
\bar{d}_{L}(\bar{z})=\bar{d}_{L}(z)\left[1-\kappa_{v}\right],
$$

where by considering the GR corrections described by: $\frac{4}{4}$

$$
\kappa_{v}=\left(\frac{c(1+z)}{\chi(z) H(z)}-1\right) \frac{z_{p}}{1+z_{p}} .
$$

We rewrite relation $(20)$ in distance modulus form:

$$
\bar{\mu}(\bar{z})=\bar{\mu}(z)+5 \log \left[1-\kappa_{v}\right] .
$$

One observes the distance modulus, $\mu(z)$, of a standard candle at observed redshift $z$ and assumes that the measured luminosity belongs to the non-distorted redshift $\bar{z}$ and put $\mu(z)=\bar{\mu}(\bar{z})$ (redshift distortion does not affect the background luminosity of the source). We hence re-write the relation $(22)$ as:

$$
\Delta \mu(z)=5 \log \left[1-\kappa_{v}\right]
$$

where $\Delta \mu(z)=\mu(z)-\bar{\mu}(z)$ is the deviation of the observed luminosity from the background model at the observed redshift $z$.

It is interesting to note that for a given cosmology and a given peculiar velocity, $\kappa_{v}$ changes sign at redshift $z_{*} \sim 1.5$ At redshifts $z<z_{*}$, if the source is moving toward us it is observed to be fainter than what model predicts. This will be inverse for the same object at redshifts $z>z_{*}$. In general, depending on the source redshift and the direction of the source along the line of sight, the source will look brighter or fainter than what the model predicts. Since this effect acts like (de)lensing, it is called Doppler lensing. ${ }^{27}$ By observing the distance modulus $\mu(z)$ and computing $\Delta \mu=\mu-\bar{\mu}$, the parameter $\kappa_{v}$ and the peculiar velocity are computed by using equation (23) and (21). One can show that relation (18) is the approximate version of relation (20) at low redshifts. In Fig. 2, the solid curve demonstrates relation 230. The distance modulus of the data points are given by Union 2 SNe Ia catalog 37 for $z<0.2 . \bar{\mu}$ is computed for $\Omega_{m}=0.27, \Omega_{\Lambda}=0.73$ and $H_{0}=70 \mathrm{~km} / \mathrm{s} / \mathrm{Mpc}$. The coloured symboles represent peculiar velocities computed from relation (18). We have substituted the non-relativistic $v_{p}$ in relation (21) and plotted $\Delta \mu$ vs. $\kappa_{v}$. The non-relativistic approximation overestimates the absolute value of the peculiar velocities. The overestimation increases by redshift.

We can compare the variation of $\kappa_{g}$ and $\kappa_{v}$ as function of redshift in Fig. 1 It shows that at low redshift the lensing effect is subdominant and the luminosity deviation from the background model is caused mainly by the peculiar velocities.

To include the effect of changes in gravitational potentials on the luminosity and redshift measurement, we substitute equation (12) in equation (8). By taking into account the relations $(9)$ and $(14)$, we obtain: ${ }^{27}$

$$
D_{L}(z)=\bar{d}_{L}(z)\left[1-\kappa_{v}-\kappa_{g}-\kappa_{S W}-\kappa_{I S W}\right]
$$

where $D_{L}(z)$ is the observed luminosity distance at observed redshift $z$, and

$$
\kappa_{S W}(z)=2 \Phi_{s}(z)+\frac{c(1+z)}{2 H(z) \chi(z)} z_{S W}
$$




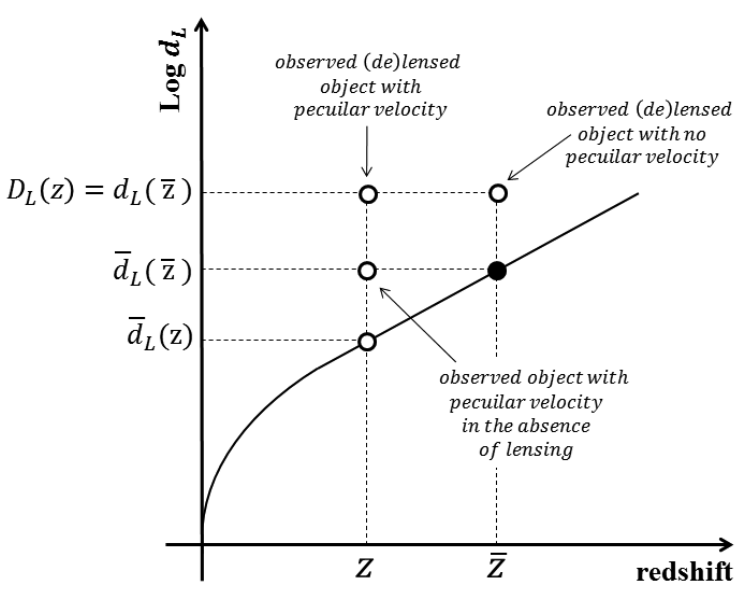

Fig. 3. Schematic view of the effect of peculiar velocities and gravitational lensing on the background cosmology curve (solid curve). A standard candle, located originally at redshift $\bar{z}$ with unperturbed luminosity distance $\bar{d}_{L}(\bar{z})$, will be measured at a different redshift $(z)$, due to peculiar velocity, and different luminosity distance $\left(D_{L}\right)$ due to (de)lensing effect. At redshift $z$, we expect a standard candle to have (unperturbed) luminosity distance $d_{L}(z)$.

$$
\kappa_{I S W}(z)=\frac{2}{\chi_{s}(z)} \int_{0}^{\chi_{s}(z)} d \chi \Phi+\frac{2}{c}\left(1+\frac{c(1+z)}{H(z) \chi(z)}\right) \int_{0}^{\chi_{s}(z)} d \chi \Phi^{\prime} .
$$

The last equation shows the contribution of the Integrated Sachs-Wolf effect on the luminosity fluctuation. This term contributes more for sources located at high redshifts. At low and intermediate redshifts $(z<2)$, we neglect $\kappa_{S W}$ and $\kappa_{I S W}$.

\subsection{Luminosity distance with peculiar velocity in a clumpy universe}

Consider an unperturbed FRW Universe where the luminosity distances of the celestial objects are described by equation (4). For the sources located at intermediate redshift, the existence of voids and clumps can perturb equation (4) in two ways: 1. The lensing effect of line-of-sight structures keeps the comoving redshift unchanged and changes the source background luminosity, through relation (11).

2. The peculiar velocity, induced locally by nearby structures of the host galaxy, keeps the (de)lensed luminosity unchanged and perturbs the comoving redshift from $\bar{z}$ to $z$ (Doppler lensing) through relation $(20)$. These effects are schematically shown in Fig. 3 Consider an object located at comoving redshift $\bar{z}$ with background luminosity distance $\bar{d}_{L}(\bar{z})$. We observe the same object at redshift $z$ and luminosity distance $D_{L}(z)$. From Fig. 3 , we have $D_{L}(z)=d_{L}(\bar{z})$ (lensing is the only cause of (de)magnifying the background luminosity). By neglecting the SW and ISW effects, we will have:

$$
D_{L}(z)=\bar{d}_{L}(z)\left[1-\kappa_{v}-\kappa_{g}\right],
$$

We now have all the elements to compute the peculiar velocity at the observed 
redshift $z$ :

$$
v_{p} / c=\left(1-\kappa_{g}-\frac{D_{L}(z)}{\bar{d}_{L}(z)}\right)\left(\frac{c(1+z)}{\chi(z) H(z)}-1\right)^{-1}
$$

For $\kappa_{g}=0$, we arrive at equation 23) where $\mu(z)=5 \log D_{L}(z)+25$. Measuring the peculiar redshift from the observed $D_{L}(z)$ (or $\mu(z)$ ) by the method described in the previous section ( Eq. 23) will obviously overestimate the peculiar velocity. This is because by assuming $\mu(z)=\bar{\mu}(\bar{z})$, we are attributing all of the deviation from the Hubble diagram to redshift-space distortion, neglecting the gravitational (de)lensing.

A numerical example of velocity overestimation can be given as follow. It is possible to have a convergence factor $\kappa_{g} \sim 0.1$ at redshift $\bar{z} \sim 1$. This convergence is equivalent to distance modulus excess $|\Delta \mu| \sim 0.2$. If one attributes this luminosity excess to peculiar velocity by using Eq. 23, we end up with $v_{p} \sim 10,000 \mathrm{~km} / \mathrm{s}$ which is unrealistic.

From observational point of view, we can not yet easily separate the contribution of the peculiar velocity and gravitational (de)lensing. A profound map for $\kappa_{g}$ is needed to estimate the (de)lensing effect at intermediate redshifts. This map is not yet available. Moreover, the presence of the systematic errors and uncertainties on the luminosity measurement of the standard candles can mimic the observed luminosity residuals of the Hubble diagram.

To compute the lensing map, the density contrast field, $\delta_{m}$, in equation 10 p should be integrated along the line of sight up to the intermediate redshifts. The method is explained in ${ }^{9}$ to compute $\kappa_{g}$ map with $z_{\max }=0.04$ for SDSS DR10. Assuming a unity bias between the CDM and luminous matter, $\delta_{m}$ can be estimated by measuring the luminosity contrast of galaxies, residing in over and under dense regions. Regarding the void galaxies, Tavasoli et a ${ }^{39}$ explained how to derive the void catalogues and compute the density contrast by applying a $3 \mathrm{D}$ grid on a volume-limited galaxy sample. One of the parameters that affects the precision on $\kappa_{g}$ measurement is the spatial resolution of the derived density contrast field.

According to Union 2 compilation paper $\frac{37}{37}$ one of the main sources of the intrinsic dispersion in distance modulus measurement is the 0.15 magnitudes error assigned to SNe Ia of the whole sample to decrease the weight of the poorer-measured (sub)samples. Regarding other systematics, they have shown that the propagation of the calibration uncertainties of the photometric passbands on the measured distance moduli should be taken into account for each SNe Ia sample separately. This can add uncertainties up to 0.04 magnitudes depending on the sample and the passband. Considering the photometric and spectroscopic diversity of the SNe Ia for wavelengths shorter than rest-frame B-band, they included 0.03 magnitudes correlated uncertainty for all $\mathrm{SNe}$ Ia with photometric band bluer than rest-frame $3500 \AA$. This affects more the SN Ia at higher redshifts. Other sources of uncertainties such as Malmquist bias, Galactic and intergalactic extinction are included as well but they have smaller contributions. Regarding all these facts, we analyse the luminosity residuals of the Hubble diagram as follow:

Figure 4 shows the absolute value of the computed (radial) peculiar velocity versus redshift for SNe Ia from Union 2 catalog. We have ignored any probable lensing effect. The velocities are hence computed from relations $(23),(21)$ and (15). 


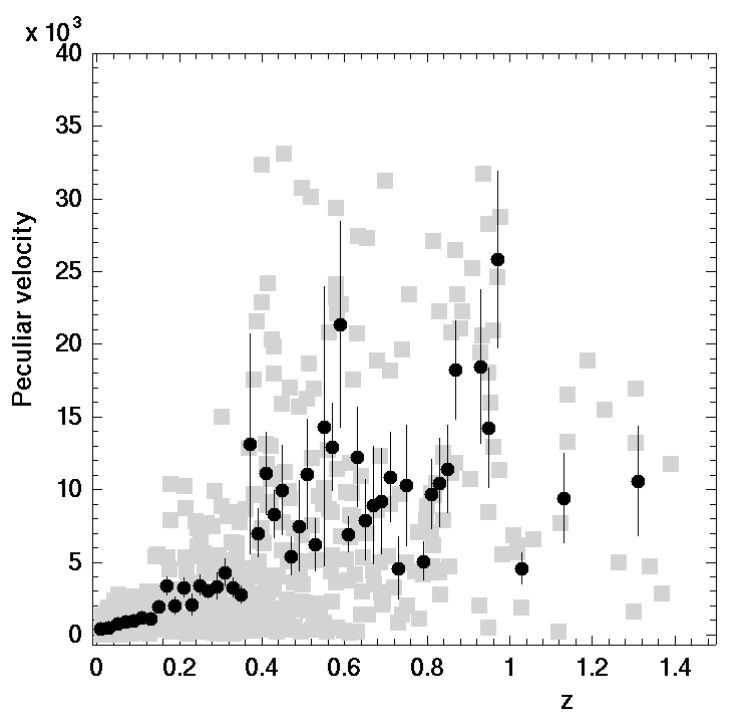

Fig. 4. The absolute values of the peculiar velocities $(\mathrm{km} / \mathrm{s})$, computed from relation $23 \mathrm{up}$ to $z=1.4$ for SNe Ia of Union2 catalog. The data (grey boxes) are averaged with bin width of 0.01 redshift (black circles). The error bars show one sigma scatter per bin. No correction due to lensing effect is applied.

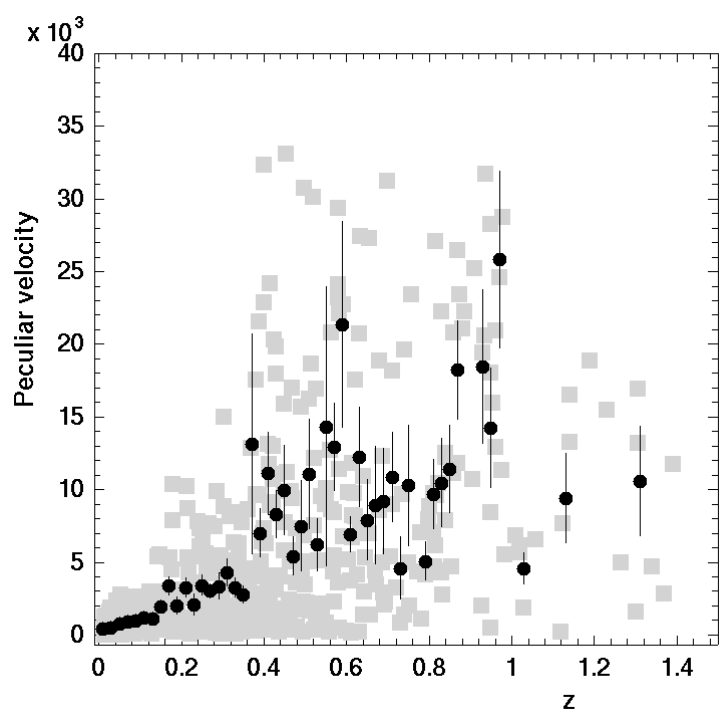

Fig. 5. Similar to Fig. 4 but the velocities $(\mathrm{km} / \mathrm{s})$ are computed from the uncertainties on luminosity measurements rather than the luminosity residuals $\mu-\bar{\mu}$.

As can be illustrated, the velocities are $\sim 1000 \mathrm{~km} / \mathrm{s}$ for $z<0.15$ and then increase dramatically with $z$ for higher redshifts. Figure 5 shows the peculiar velocities pro- 
duced by the uncertainties of the observed distance moduli for Union 2 catalog. This is done by replacing $\Delta \mu(z)$ in relation $(23)$ by the uncertainties on the $\mathrm{SNe}$ distance moduli and computing the corresponding velocity for each SN. In both figures, each grey box represents a SN Ia. Black circles show the averaged velocities in redshift bin of 0.01 . The error bars correspond to one sigma scatter per bin. We should mention that the number of SNe Ia per bin decreases by redshift. As can be seen, both figures 4 and 5 show the same trends. This is equivalent to say that the uncertainties on SNe Ia luminosity are still large-enough that the deviations from the background $(\Lambda \mathrm{CDM})$ model are not statistically significant. This fact prevents us from attributing the velocity excess at higher redshifts to the lensing effect. Furthermore, even for $z<0.15$, the velocities produced by pure uncertainties on the observed distance moduli are larger than the one produced by $\Delta \mu(z)$. This shows that the peculiar velocity measurements are not sufficiently precise due to, once again, the large uncertainties on SNe Ia luminosity measurement.

\section{Conclusion and future prospects}

Considering the FRW metric and the $\Lambda$ CDM model, one can obtain the luminosity distance as a function of the cosmological redshift. At low redshifts, the deviation of the observed luminosity distance from the model prediction is attributed to the source peculiar velocity induced by local structures around the source. The peculiar velocity is obtained by computing the amount of difference in the luminosity distance in the $\Lambda \mathrm{CDM}$ model. The peculiar velocity measurements are in the heart of modern cosmology to probe the local universe and also to study the cosmological models in large scales. In this work we investigate the peculiar velocity measurement in perturbed-FRW universe and we assert and show that relativistic corrections are important in our results and interpretation. In one hand, one should calculate the velocities with a relativistic doppler effect, on the other hand we should take into account the effects of lensing, Sachs-Wolfe (the main and integrated one) and the peculiar velocity of the source and observer. In this work we investigate the interplay of the peculiar velocity and lensing at low and specially at intermediate redshifts. We also want to bring the attention of the community to the fact that using less model-dependent methods, such as linear theory, can help us to derive the peculiar velocity by considering the matter distribution around the source. Such methods lead us to reexamine the cosmological models according to model predictions for the peculiar velocities. $\frac{9}{1 n}$ this work, we showed that as we approach to intermediate redshifts, the lensing effect of the line-of-sight structures gets stronger. The deviations of the luminosity distance from the model, caused by gravitational convergence, become comparable to the effect of peculiar velocities. Therefore, to compute the peculiar velocity, one should firstly correct the luminosity distance for the (de)lensing effect. We showed that with the present data of the standard candles, using the Union 2 data sample, it is not yet possible to extract the contribution of the peculiar velocity and the gravitational lensing on the luminosity deviation from the background $\Lambda \mathrm{CDM}$ model. This is essentially due to the rather large (systematic) uncertainties in SNe Ia data. These uncertainties prevent us from precise measurement of the peculiar velocity even at low redshifts. However, including the effect of cosmic convergence on peculiar velocities is crucial for future surveys (such 
as LSST ${ }^{35}$ and Euclid $\sqrt{36}$ ). These surveys will map cosmic structures up to intermediate redshifts where gravitational lensing plays an important role. Finally worth to mention again that the extension of this work with tilted coordinates ${ }^{33}$ can have new prospects on the relativistic effects study in large scale structure observations.

\section{Acknowledgements}

We would like to thank Joseph Silk, Roya Mohayaee and Marc Moniez for their insightful comments and useful discussions. We thank R. Mansouri and S.M. Mahdavi for facilitating the stay of FH at Sharif University of Technology. The research of $\mathrm{FH}$ at IAP is supported by a grant from the International Balzan Foundation via the Oxford New College-Johns Hopkins University Center for Cosmological Studies.

\section{References}

1. A. G. Riess et al. [Supernova Search Team Collaboration], "Observational evidence from supernovae for an accelerating universe and a cosmological constant," Astron. J. 116, 1009 (1998) astro-ph/9805201.

2. S. Perlmutter et al. [Supernova Cosmology Project Collaboration], "Measurements of Omega and Lambda from 42 high redshift supernovae," Astrophys. J. 517, 565 (1999) astro-ph/9812133.

3. M. Bartelmann and P. Schneider, "Weak gravitational lensing," Phys. Rept. 340, 291 (2001) astro-ph/9912508.

4. C. Bonvin, R. Durrer and M. A. Gasparini, "Fluctuations of the luminosity distance," Phys. Rev. D 73, 023523 (2006) [Phys. Rev. D 85, 029901 (2012)] doi:10.1103/PhysRevD.85.029901, 10.1103/PhysRevD.73.023523 astro-ph/0511183.

5. T. M. Davis, L. Hui, J. A. Frieman, T. Haugbolle, R. Kessler, B. Sinclair, J. Sollerman and B. Bassett et al., "The effect of peculiar velocities on supernova cosmology," Astrophys. J. 741, 67 (2011) arXiv:1012.2912 [astro-ph.CO]].

6. J. Koda, C. Blake, T. Davis, C. Magoulas, C. M. Springob, M. Scrimgeour, A. Johnson and G. B. Poole et al., "Are peculiar velocity surveys competitive as a cosmological probe?," arXiv:1312.1022 [astro-ph.CO].

7. S. Baghram, M. S. Movahed and S. Rahvar, Phys. Rev. D 80, 064003 (2009) doi:10.1103/PhysRevD.80.064003 arXiv:0904.4390 [astro-ph.CO]].

8. S. Baghram and S. Rahvar, "Structure formation in $f(R)$ gravity: A distinguishing probe between the dark energy and modified gravity," JCAP 1012, 008 (2010) doi:10.1088/1475-7516/2010/12/008 arXiv:1004.3360 [astro-ph.CO]].

9. S. Baghram, S. Tavasoli, F. Habibi, R. Mohayaee and J. Silk, "Unraveling the nature of Gravity through our clumpy Universe," arXiv:1411.7010 [astro-ph.CO].

10. S. Khosravi, A. Mollazadeh and S. Baghram, "ISW-galaxy cross correlation: a probe of dark energy clustering and distribution of dark matter tracers," JCAP 1609, no. 09, 003 (2016) doi:10.1088/1475-7516/2016/09/003 arXiv:1510.01720 [astro-ph.CO]].

11. N. Kaiser, "Theoretical implications of deviations from Hubble flow," Mon. Not. Roy. Astron. Soc. 231, 149 (1989).

12. A. Dekel, "Dynamics of cosmic flows," Ann. Rev. Astron. Astrophys. 32, 371 (1994) astro-ph/9401022.

13. M. A. Strauss and J. A. Willick, "The Density and peculiar velocity fields of nearby galaxies," Phys. Rept. 261, 271 (1995) astro-ph/9502079.

14. K. L. Masters, C. M. Springob, M. P. Haynes and R. Giovanelli, "SFI++ I: A New 
I-band Tully-Fisher Template, the Cluster Peculiar Velocity Dispersion and H0," Astrophys. J. 653, 861 (2006) astro-ph/0609249.

15. D. Sarkar, H. A. Feldman and R. Watkins, "Bulk flows from velocity field surveys: A Consistency check," Mon. Not. Roy. Astron. Soc. 375, 691 (2007) astro-ph/0607426.

16. A. Abate, S. Bridle, L. F. A. Teodoro, M. S. Warren and M. Hendry, "Peculiar Velocities into the Next Generation: Cosmological Parameters From Large Surveys without Bias from Nonlinear Structure," arXiv:0802.1935 [astro-ph].

17. G. Lavaux, R. Mohayaee, S. Colombi, R. B. Tully, F. Bernardeau and J. Silk, "Observational biases in Lagrangian reconstructions of cosmic velocity fields," arXiv:0707.3483 [astro-ph]].

18. A. Nusser and M. Davis, "The cosmological bulk flow: consistency with $\Lambda$ CDM and $z \approx 0$ constraints on $\sigma_{8}$ and $\gamma$," Astrophys. J. 736, 93 (2011) arXiv:1101.1650 [astroph.CO]].

19. R. B. Tully and J. R. Fisher, "A New method of determining distances to galaxies," Astron. Astrophys. 54, 661 (1977).

20. J. L. Tonry, A. Dressler, J. P. Blakeslee, E. A. Ajhar, A. B. Fletcher, G. A. Luppino, M. R. Metzger and C. B. Moore, "The sbf survey of galaxy distances. 4. Sbf magnitudes, colors, and distances," Astrophys. J. 546, 681 (2001) astro-ph/0011223.

21. S. M. Faber and R. E. Jackson, "Velocity dispersions and mass to light ratios for elliptical galaxies," Astrophys. J. 204, 668 (1976).

22. W. L. Freedman et al. [HST Collaboration], "Final results from the Hubble Space Telescope key project to measure the Hubble constant," Astrophys. J. 553, 47 (2001) astro-ph/0012376.

23. D. E. Holz and E. V. Linder, "Safety in numbers: Gravitational lensing degradation of the luminosity distance-redshift relation," Astrophys. J. 631, 678 (2005) astro$\mathrm{ph} / 0412173$.

24. S. Hilbert, J. Hartlap, S. D. M. White and P. Schneider, "Ray-tracing through the Millennium Simulation: Born corrections and lens-lens coupling in cosmic shear and galaxy-galaxy lensing," Astron. Astrophys. 499, 31 (2009) arXiv:0809.5035 [astro$\mathrm{ph}]$.

25. I. Ben-Dayan, M. Gasperini, G. Marozzi, F. Nugier and G. Veneziano, "Average and dispersion of the luminosity-redshift relation in the concordance model," JCAP 1306, 002 (2013) arXiv:1302.0740 [astro-ph.CO]].

26. K. Bolejko and P. G. Ferreira, "Ricci focusing, shearing, and the expansion rate in an almost homogeneous Universe," JCAP 1205, 003 (2012) arXiv:1204.0909 [astroph.CO]].

27. D. J. Bacon, S. Andrianomena, C. Clarkson, K. Bolejko and R. Maartens, "Cosmology with Doppler Lensing," arXiv:1401.3694 [astro-ph.CO].

28. T. M. Davis and M. I. Scrimgeour, "Deriving accurate peculiar velocities (even at high redshift)," arXiv:1405.0105 [astro-ph.CO].

29. Y. -Z. Ma and D. Scott, "Velocities hasten to tell us about the Universe," Astron. Geophys. 55, 3 (2014) arXiv:1406.1136 [astro-ph.CO]].

30. G. F. R. Ellis and C. G. Tsagas, "A Relativistic approach to nonlinear peculiar velocities and the Zeldovich approximation," Phys. Rev. D 66, 124015 (2002) doi:10.1103/PhysRevD.66.124015 astro-ph/0209143.

31. C. G. Tsagas, "Large-scale peculiar motions and cosmic acceleration," Mon. Not. Roy. Astron. Soc. 405, 503 (2010) doi:10.1111/j.1365-2966.2010.16460.x arXiv:0902.3232 [astro-ph.CO]].

32. C. G. Tsagas, "Peculiar motions, accelerated expansion and the cosmological axis," Phys. Rev. D 84, 063503 (2011) doi:10.1103/PhysRevD.84.063503 arXiv:1107.4045 
[astro-ph.CO]].

33. C. G. Tsagas and M. I. Kadiltzoglou, "Deceleration parameter in tilted Friedmann universes," Phys. Rev. D 92, no. 4, 043515 (2015) doi:10.1103/PhysRevD.92.043515 arXiv:1507.04266 [gr-qc]].

34. R. K. Sachs and A. M. Wolfe, "Perturbations of a cosmological model and angular variations of the microwave background," Astrophys. J. 147, 73 (1967) [Gen. Rel. Grav. 39, 1929 (2007)].

35. P. A. Abell et al. [LSST Science and LSST Project Collaborations], "LSST Science Book, Version 2.0," arXiv:0912.0201 [astro-ph.IM].

36. L. Amendola et al. [Euclid Theory Working Group Collaboration], "Cosmology and fundamental physics with the Euclid satellite," Living Rev. Rel. 16 (2013) 6 arXiv:1206.1225 [astro-ph.CO]].

37. R. Amanullah, C. Lidman, D. Rubin, G. Aldering, P. Astier, K. Barbary, M. S. Burns and A. Conley et al., Spectra and Light Curves of Six Type Ia Supernovae at 0.511 z 1.12 and the Union2 Compilation, Astrophys. J. 716, 712 (2010) arXiv:1004.1711 [astro-ph.CO]].

38. H. Guo, Z. Zheng, I. Zehavi, P. S. Behroozi, C. H. Chuang, J. Comparat, G. Favole and S. Gottloeber et al., "Redshift-Space Clustering of SDSS Galaxies - Luminosity Dependence, Halo Occupation Distribution, and Velocity Bias," arXiv:1505.07861 [astro-ph.CO].

39. S. Tavasoli, H. Rahmani, H. G. Khosroshahi, K. Vasei, M. D. Lehnert, "The Galaxy Population in Voids: Are All Voids the Same?" Astrophys. J. 803L, 13T (2015)

40. J. Aikio, P. Màhönen, "A Simple Void-searching Algorithm" Astrophys. J. 497, 534A (1998) 